\title{
Complications of Embolization for Cerebral Arteriovenous Malformations
}

\author{
K. SUGIU, K. TOKUNAGA, W. SASAHARA, K. WATANABE, A. NISHIDA, S. ONO, \\ S. NISHIO, I. DATE, D. A. RÜFENACHT* \\ Department of Neurological Surgery, Okayama University Medical School, Okayama; Japan \\ * Section of Neuroradiology, Department of Radiology, University Hospital of Geneva, Geneva; Switzerland
}

Key words: arteriovenous malformation, brain, embolization, complication

\section{Summary}

Embolization is recognized as an important adjunct in the treatment of cerebral arteriovenous malformations (AVMs). We reviewed our results of embolizations for AVMs and discussed procedure-related complications. Eleven complications were recorded in 68 consecutive patients (16\%). Of these, four were technical problems including a glued catheter, inability to withdraw the catheter, vessel perforation by the microcatheter, and coil migration. Other complications included three cases of ischemic symptoms due to retrograde thrombosis, two cases of asymptomatic cerebral infarction, one case of asymptomatic small haemorrhage due to venous occlusion, and one case of post-embolization haemorrhage of unknown etiology. Our morbidity rate was $7 \%$, mortality rate was $0 \%$, and asymptomatic complication rate was $9 \%$, retorospectively. Further improvements to endovascular techniques and devices are required.

\section{Introduction}

Since the first modern report of endovascular embolization for cerebral arteriovenous malformations (AVMs) by Luessenhop in $1960^{1}$, there has been continuing development concerning both endovascular devices and techniques. The results of endovascular treatment have been im- proved. However, the successful treatment of AVMs remains a challenge because of its higher treatment morbidity and mortality rate, which reported between $4 \%$ and $39 \%$ in the literature ${ }^{2-8}$. We reviewed our results of embolization for AVMs and focused on its complications.

\section{Clinical Material and Methods}

\section{Patient population}

Between December 1996 and September 2003, 68 patients with cerebral AVMs underwent trans-arterial embolization by K.S. or K.T. as a main operator. Fifty-one patients were treated at Okayama University Hospital and 17 were treated at Geneva University Hospital where K.S. and K.T. were stayed for endovascular training. Our series consisted of 46 male and 22 female patients, whose ages ranges from 11 to 75 years (median age 41years). Thirty-five patients $(51 \%)$ presented with haemorrhage, 24 with seizure (35\%), three with ischemic symptoms $(4 \%)$, one with neuralgia $(1 \%)$, and five were incidentally found asymptomatic patients (7\%). The Spetzler-Martin grade was I in nine patients $(13 \%)$, II in $23(34 \%)$, III in $28(41 \%)$, IV in $8(12 \%)$. No patient had an AVM of grade $\mathrm{V}$ in this series. The aim of embolization was complete-cure-aimed embolization in 12 
patients $(18 \%)$, pre-operative embolization in $44(64 \%)$, pre-stereotactic radiosurgical embolization in $12(18 \%)$.

\section{Embolization technique}

Patients underwent endovascular embolization with systemic heparinization while under local anesthesia. All procedures were performed with the standard femoral artery approach. A flow-guided micocatheter and liquid embolic materials were used for the completecure-aimed or pre-stereotactic radiosurgical embolization, although various microcatheters and embolic materials were used for pre-operative embolization. For the embolization of the eloquent area, provocative test was performed. Aggressive adjuvant techniques such as intensive hypotension or temporary cardiac arrest were not used.

\section{Results}

Sixty-eight consecutive patients were embolized in this series. The number of treatment sessions per patients was from one to six times (average 1.4). The types of the microcatheter used in these procedures were flow-guided type in 38 patients, and over-the-wire type in 30 patients. Liquid embolic materials were used in 47 patients, while solid embolus were used in 22 patients. In one patient, both liquid and solid materials were used. Liquid materials included N-butyl cyanoacrylate (NBCA), cellulose acetate polymer (CAP), and ethylene-vinyl alcohol copolymer $(\mathrm{EVOH})$. Solid materials included polyvinyl alcohol (PVA) particles, trisacryl gelatin microspheres, silk threads, and liquid coils.

Complications were recorded in 11 patients (16\%). They were counted two in 12 completecure-aimed embolization (17\%), eight in 44 pre-operative embolization $(18 \%)$, and one in 12 pre-stereotactic radiosurgical embolization (8\%). Five complications $(7 \%)$ remained permanent neurological deficits, although six (9\%) were transient or asymptomatic complications. No mortality was recorded in this study.

Microcatheter related complications were found two $(5 \%)$ in 38 flow-guided catheter used group, and one $(3 \%)$ in over-the wire catheter used group. The former included one case of glued catheter and one case of inability to withdraw catheter due to vasospasm, both of which were surgically removed without any complications. The latter included a case of feeding artery perforation by the microcatheter resulted in left hemiparesis.

Embolic material related complications were found five cases (11\%) in 47 liquid used group, and one $(5 \%)$ in solid used group. The former included one case of glued catheter (same case as above), three cases of ischemic symptoms due to post-embolization retrograde arterial thrombosis, and post-embolization small haemorrhage due to venous occlusion. Three patients who had ischemic complications showed permanent neurological deficits, while other two patients showed no neurological symptoms. The latter included a case of migration of the liquid coil into the normal artery caused no symptoms.

In two cases, we intentionally embolized normal arteries with vascular malformations resulted in asymptomatic small cerebral infarction. In these two cases, it was impossible to catheterize into the nidus super-selectively, we decided to embolize both AVM and silent area of the brain. One patient had haemorrhage around feeding artery three hours after complete obliteration of AVM located on right motor cortex. We could not find the reason of haemorrhage.

In summary, eleven complications were detected in 68 patients $(16 \%)$. Of these, four were technical problems including glued catheter, inability to withdraw catheter, vessel perforation by the microcatheter, and migration of coil. Other complications included three cases of ischemic symptom due to retrograde thrombosis, two cases of asymptomatic cerebral infarction, one case of asymptomatic small haemorrhage due to venous occlusion, and one case of post-embolization haemorrhage of unknown etiology.

\section{Discussion}

Although not without complications, endovascular embolization has been thought to be an important adjunct in the treatment of cerebral AVMs ${ }^{3,5,6}$. Pre-surgical embolization is induced to enhance the safety of surgery by occluding the AVM nidus ${ }^{2,6}$. Pre-stereotactic radiosurgical embolization is also useful in reduction of AVM volume, occlusion of aneurysms, and obliteration of high-flow fistulae ${ }^{9}$. Complete obliteration of AVM can be sometimes achieved by embolization alone ${ }^{4}$. 
Reported complication rates of AVM embolization are varied. Valvanis et $\mathrm{Al}^{4}$ reported $5.1 \%$ of morbidity and $1.3 \%$ of mortality rate in their series of 387 patients with AVMs. Wikholm et $\mathrm{Al}^{3}$ reported approximately $40 \%$ complication rate in their 150 patients series. Although this percentage is thought to be extremely high, they also described that the combined risk of death and severe complications amounts to $8.0 \%$ of patients. They concluded this percentage is approximately equal to 3.2 years of natural history mortality / morbidity.

In our eleven cases having complications, there are four technical problems, five cases of cerebral infarction, two cases of asymptomatic cerebral infarction, one case of asymptomatic small haemorrhage due to venous occlusion, and one case of post-embolization haemorrhage of unknown etiology. From a technical

\section{References}

1 Luessenhop AJ, Spence WT: Artificial embolization of cerebral arteries. Report of use in a case of arteriovenous malformation. JAMA 172: 1153-1155, 1960.

2 Purdy PD, Batjer HH et Al: Management of haemorrhagic complications from preoperative embolization of arteriovenous malformations. J Neurosurg 74: 205-211, 1991.

3 Wikholm G, Lundqvist $\mathrm{C}$ et $\mathrm{Al}$ : Embolization of cerebral arteriovenous malformations: Part I-Technique, morphology, and complications. Neurosurgery 39: 448459, 1996.

4 Valvanis A, Yasargil MG: The endovascular treatment of brain arteriovenous malformations. Adv Tech Stand Neurosurg 24: 131-214, 1998.

5 Paulsen RD, Steinberg GK et Al: Embolization of basal ganglia and thalamic arteriovenous malformations. Neurosurgery 44: 991-997, 1999.

6 Song JK, Eskridge JM et Al: Preoperative embolization of cerebral arteriovenous malformations with silk sutures: analysis and clinical correlation of complications revealed on computerized tomography scanning. J Neurosurg 92: 955-960, 2000.

7 Jahan R, Murayama Y et Al: Embolization of arteriovenous malformations with Onyx: clinicopathological experience in 23 patients. Neurosurgery 48: 984-997, 2001. point of view, atraumatic superselective microcatheterization and adequate injection of the embolic materials are desired. These require manual skills, knowledge of anatomy and devices, and experiences. In five cases of cerebral infarction, three have had delayed neurological deficits due to retrograde thrombosis. An adequate systemic heparinization after successful embolization will be needed in such cases.

\section{Conclusions}

Our results indicated a $16 \%$ complication rate in the series of 68 patients. Morbidity rate was $7 \%$, mortality rate was $0 \%$, and asymptomatic complication rate was $9 \%$. Further improvements to endovascular techniques and devices are required for better patient outcome.
8 Taylor CL, Dutton K et Al: Complications of preoperative embolization of cerebral arteriovenous malformations. J Neurosurg 100: 810-812, 2004.

9 Gobin YP, Laurent A et Al: Treatment of brain arteriovenous malformations by embolization and radiosurgery. J Neurosurg 85: 19-28, 1996. 